\title{
On Artin L-Functions for Octic Quaternion Fields
}

\author{
Sami Omar
}

\section{CONTENTS}

1. Introduction

2. Definitions and Notation

3. Quaternion Extensions

4. Computation of $n_{\chi}$

5. An Upper Bound for $n_{\chi}$ and Low Zeros of $L(s, \chi)$

6. Computations of $n_{\chi}$ for Quaternion Fields

Acknowledgments

References
AMS Subject Classification: 11R42

Keywords: Artin $L$-functions, zeros, quaternion fields
We study the Artin L-function $\mathrm{L}(\mathrm{s}, \chi)$ associated to the unique character $\chi$ of degree 2 in quaternion fields of degree 8 . We first explain how to find examples of quaternion octic fields with not too large a discriminant. We then develop a method yielding a quick computation of the order $\mathrm{n}_{\chi}$ of the zero of $\mathrm{L}(\mathrm{s}, \chi)$ at the point $\mathrm{s}=\frac{1}{2}$. In all our calculations, we find that $\mathrm{n}_{\chi}$ only depends on the sign of the root number $W(\chi)$; indeed $n_{\chi}=0$ when $\mathrm{W}(\chi)=+1$ and $\mathrm{n}_{\chi}=1$ when $\mathrm{W}(\chi)=-1$. Finally we give some estimates on $n_{\chi}$ and low zeros of $L(s, \chi)$ on the critical line in terms of the Artin conductor $\mathfrak{f}_{\chi}$ of the character $\chi$.

\section{INTRODUCTION}

The well known conjecture that the zeros of the Riemann zeta function are simple can be also stated for a more general class of Dirichlet $L$-series and Artin $L$-functions associated to one-dimensional characters of number fields. Conjecturally when the base field is $\mathbb{Q}$, these functions never vanish at the central critical point [Murty and Murty 1997]. More particularly, a question of J.-P. Serre is to know whether the order $n_{\chi}$ of a zero of $L(s, \chi)$ at the point $s=\frac{1}{2}$ is the smallest possible with respect to the constraints imposed by the properties of the character $\chi$, in particular those imposed by the sign of the root number $W(\chi)$ when $\chi$ is real-valued.

A precise form of this conjecture is stated in [Goss 1996, p. 324]. In this paper, we study the case of two-dimensional characters $\chi$ arising from quaternion fields $N / \mathbb{Q}$ of degree 8 . Recall that the explicit computation of values of Artin $L$-functions done in [Tollis 1997] based on a formula due to A. F. Lavrik and E. Friedman (see [Cohen 2000, Section 10.3]) becomes very lengthy from degree 7 onwards. The expected running time is roughly $O\left(\sqrt{\mathfrak{f}_{\chi}}\right)$. However, for the method we develop here, the required time is $O\left(\ln \mathfrak{f}_{\chi}\right)$, which allows us to deal with degree-eight fields. We also give faster 
algorithms that depend on the Generalized Riemann Hypothesis.

\section{DEFINITIONS AND NOTATION}

Let $N / K$ be a Galois extension of a number field with Galois group $G=\operatorname{Gal}(N / K)$, let $(\rho, V)$ be a representation of $G$ and $\chi$ its character. Then the Artin $L$-function attached to $\chi$ is defined:

$$
L(N / K, \chi, s)=\prod_{\mathfrak{p} \text { finite }} \frac{1}{\operatorname{det}\left(1-\rho\left(\varphi_{\mathfrak{P}}\right) \mid V^{I_{\mathfrak{P}}} N(\mathfrak{p})^{-s}\right)},
$$

where the product is over all finite primes $\mathfrak{p}$ of $K$. Here $\varphi_{\mathfrak{P}}$ is the Frobenius automorphism of one $\mathfrak{P}$ above an unramified $\mathfrak{p}$. For ramified $\mathfrak{p}$, see [Martinet 1977]. The Artin $L$-series converges uniformly in half-planes $\operatorname{Re} s>1+\delta$ (with $\delta>0$ ) and defines an analytic function on the half-plane $\operatorname{Re} s>1$. Using basic properties of representations, one can prove that

$$
\zeta_{N}(s)=\zeta_{K}(s) \prod_{\chi \neq 1} L(N / K, \chi, s)^{\chi(1)},
$$

where $\chi$ varies over the nontrivial irreducible characters of $G$. The positive integer $\chi(1)$ arises from the decomposition of the the regular representation $\operatorname{reg}_{G}$ of $G$ into $\operatorname{reg}_{G}=\sum_{\chi} \chi(1) \chi$; see [Serre 1978].

In order to obtain an $L$-function with a functional equation, it is necessary to introduce Euler factors for the infinite primes of $K$. For every infinite place $\mathfrak{p}$ of $K$, define

$L_{\mathfrak{p}}(N / K, \chi, s)= \begin{cases}L_{\mathbb{C}}(s)^{\chi(1)}, & \mathfrak{p} \text { complex } \\ L_{\mathbb{R}}(s)^{n^{+}} L_{\mathbb{R}}(s+1)^{n^{-}}, & \mathfrak{p} \text { real },\end{cases}$

where

$$
L_{\mathbb{C}}(s)=2(2 \pi)^{-s} \Gamma(s), \quad L_{\mathbb{R}}(s)=\pi^{-\frac{s}{2}} \Gamma(s / 2)
$$

and

$$
n^{+}=\frac{\chi(1)+\chi\left(\varphi_{\mathfrak{P}}\right)}{2}, \quad n^{-}=\frac{\chi(1)-\chi\left(\varphi_{\mathfrak{P}}\right)}{2} .
$$

Define the enlarged Artin function $\Lambda(N / K, \chi, s)$ by

$$
\begin{aligned}
& \Lambda(N / K, \chi, s) \\
& \quad=c(N / K, \chi)^{s / 2} L_{\infty}(N / K, \chi, s) L(N / K, \chi, s),
\end{aligned}
$$

where

$$
c(N / K, \chi)=\left|d_{K}\right|^{\chi(1)} N_{K / \mathbb{Q}}(\mathfrak{f}(N / K, \chi))
$$

and

$$
L_{\infty}=\prod_{\mathfrak{p} \mid \infty} L_{\mathfrak{p}}(N / K, \chi, s) ;
$$

this function has a meromorphic continuation to the whole complex plane and satisfies the functional equation

$$
\Lambda(N / K, \chi, 1-s)=W(\chi) \Lambda(N / K, \bar{\chi}, s),
$$

where the root number $W(\chi)$ is a constant of absolute value 1 [Martinet 1977].

Artin's conjecture says that for every irreducible character $\chi \neq 1$, the Artin $L$-function $L(N / K, \chi, s)$ is everywhere holomorphic. In particular, the quotient $\zeta_{N} / \zeta_{K}$ should be entire, as a consequence of the Aramata-Brauer Theorem [Murty and Murty 1997]. Now if we restrict our attention to the order of the zero $n_{\chi}\left(s_{0}\right)$ at some $s_{0} \in \mathbb{C}$ of the $\operatorname{Artin} L$-functions, a few results were proved in this direction; see [Stark 1974] for example. By analogy with the conjecture on the simplicity of the zeros of the Riemann zeta function, the main question is to know whether for $\operatorname{Re} s_{0}>0$ we have $n_{\chi}\left(s_{0}\right) \leq 1$ if $\chi$ is absolutely irreducible and $K=\mathbb{Q}$.

\section{QUATERNION EXTENSIONS}

In this section we describe how to compute quaternion fields and give some properties of their associated Artin $L$-functions.

Definition 3.1. A quaternion extension of $\mathbb{Q}$ is a normal extension $N$ of $\mathbb{Q}$ with Galois group $G$ isomorphic to the quaternion group $H_{8}$ of order 8 .

The quaternion group $H_{8}$ can be written $H_{8}=\langle\sigma, \tau\rangle$ with relations $\sigma^{4}=1, \tau^{2}=\sigma^{2}$ and $\tau \sigma \tau^{-1}=\sigma^{-1}$. It possesses a unique irreducible character $\chi$ of degree 2 ; one has $\chi(1)=2, \chi\left(\sigma^{2}\right)=-2$ and $\chi(s)=0$ for $s \neq 1, \sigma^{2}$.

The field $N$ contains three quadratic subfields $k_{1}$, $k_{2}, k_{3}$ with discriminants $d_{1}, d_{2}, d_{3}$ and a biquadratic subfield $K$ with discriminant $d_{1} d_{2} d_{3}$. The theorem below allows us to know under what condition a quadratic field $k=\mathbb{Q}(\sqrt{m})$ can be embedded in a quaternion field $N$. For a general formulation, see [Witt 1936].

Theorem 3.2. Let $m$ be a squarefree integer. In order that $k=\mathbb{Q}(\sqrt{m})$ should be a quadratic subfield of some quaternion field $N$, it is necessary and 
sufficient that $m$ be positive and not congruent to $-1 \bmod 8$.

By a theorem of Gauss (see [Serre 1970] for a proof), the preceding condition on $m$ holds if and only if $m=p^{2}+r^{2}+s^{2}$ where $p, r, s$ are integers. Let $K^{\prime}=\mathbb{Q}(\sqrt{m}, i)$ with $i^{2}=-1$ and let $N^{\prime}$ be a quartic cyclic extension of $K^{\prime}$ such that $N^{\prime} / \mathbb{Q}$ is Galois. Put $\langle s\rangle=\operatorname{Gal}\left(K^{\prime} / k\right),\left\langle\tau>=\operatorname{Gal}\left(K^{\prime} / \mathbb{Q}(i)\right)\right.$, and lift them to elements $\bar{s}, \bar{\tau}$ in $\operatorname{Gal}\left(N^{\prime} / \mathbb{Q}\right)$. By cohomological considerations, we have the following proposition related to the construction of quaternion fields $N$ [Damey and Payan 1970]:

\section{Proposition 3.3}

citedam. $N \subset N^{\prime}$ if and only if $N^{\prime} / \mathbb{Q}(i)$ is a quaternion extension and $\bar{s} \bar{\tau}=\bar{\tau} \bar{s}$.

Now one can write $N^{\prime}=K^{\prime}(\sqrt[4]{\alpha})$ where $\alpha \in K^{\prime} \backslash k^{2}$, thus one can compute explicitly $N^{\prime}$ by the following theorem:

Theorem 3.4. The extension $N^{\prime} / \mathbb{Q}(i)$ satisfies the conditions of Proposition 3.3 if and only if $\alpha$ can be written

$$
\alpha=m(r+i s)^{2} \frac{p+\sqrt{m}}{p-\sqrt{m}} \frac{\lambda}{\bar{\lambda}},
$$

with

$$
\lambda \in \mathbb{Q}(\sqrt{-m}),\left(r^{2}+s^{2}\right) \lambda \bar{\lambda} \notin K^{*^{2}} .
$$

From this, we deduce easily $N$ by computing the fixed subfields of $N^{\prime}$ by any lifting of $s, \bar{s} \in G^{\prime}=$ $\operatorname{Gal}\left(N^{\prime} / \mathbb{Q}\right)$ of order 2 . Since $G^{\prime}=\mathbb{Z} / 2 \mathbb{Z} \times H_{8}$, there are 3 automorphisms in $G^{\prime}$ of order 2 , but only two of them can be a lifting of $s$ and the third one has a square root in $G^{\prime}$. Therefore one can compute easily the two quaternion subfields of $N^{\prime}$. In the last section we shall give a table of many totally real and imaginary quaternion extensions with their quadratic subfields.

Now we restrict our attention to the Artin $L$ function $L(s, \chi)$ associated to the unique character $\chi$ of degree 2 of $H_{8}$. If we write $L(s, \chi)$ in terms of Dedekind zeta functions, we have:

Proposition 3.5. Let $K$ be the quartic subfield of $N$, we have:

$$
\zeta_{N}(s)=\zeta_{K}(s) L(s, \chi)^{2}=\zeta_{K}(s) L\left(N / K, \chi^{\prime}, s\right),
$$

where $\chi^{\prime}$ is the nontrivial character associated to the quadratic extension $N / K$.
From the preceding identity, we deduce that $L(s, \chi)^{2}$ is an entire function. Since $L(s, \chi)$ is meromorphic then $L(s, \chi)$ is entire too.

In Theorems 3.6 and 3.7, we give an explicit computation of $W(\chi)$ for tamely ramified extensions (those such that 2 is not ramified in $N / \mathbb{Q}$ ). We start by defining an invariant $U_{N}$ of the quaternion extension $N$, by setting it to +1 if the ring of integers $O_{N}$ of $N$ is a free $\mathbb{Z}[G]$-module, and to -1 otherwise. The Fröhlich theorem gives the general equality:

Theorem 3.6 [Fröhlich 1972]. $W(\chi)=U_{N}$.

Set

$$
\varepsilon= \begin{cases}+1 & \text { if } N \text { is real, } \\ -1 & \text { if } N \text { is imaginary }\end{cases}
$$

In [Martinet 1971], one can find an explicit criterion to know whether $O_{N}$ is a free $\mathbb{Z}[G]$-module or not:

Theorem 3.7. $O_{N}$ is a free $\mathbb{Z}[G]$-module if and only if

$$
\varepsilon \prod_{p \mid d_{N}} p \equiv \frac{1+d_{1}+d_{2}+d_{3}}{4} \bmod 4 .
$$

A look at the functional equation of $L(s, \chi)$ shows:

Theorem 3.8. If $W(\chi)=+1$ then $n_{\chi}$ is even, If $W(\chi)=-1$ then $n_{\chi}$ is odd.

and the conjecture on $n_{\chi}$ can be expressed in the following way:

Conjecture 3.9. If $W(\chi)=+1$ then $n_{\chi}=0$, If $W(\chi)=-1$ then $n_{\chi}=1$.

\section{COMPUTATION OF $n_{\chi}$}

In this section we give an explicit method to compute $n_{\chi}$ and verify numerically Conjecture 3.9 in many cases (see Section 6). For that purpose, we use Weil's explicit formula [1972], as reformulated by K. Barner [1981] for ease of computation. One can adapt this formula to $L\left(N / K, \chi^{\prime}, s\right)$ and then evaluate the sum on the zeros of the Artin $L$-function $L(s, \chi)$ in the explicit formula.

Theorem 4.1. Let $F$ satisfy $F(0)=1$ and the following conditions:

(A) $F$ is even, continuous and continuously differentiable everywhere except at a finite number of 
points $a_{i}$, where $F(x)$ and $F^{\prime}(x)$ have only a discontinuity of the first kind, such that $F\left(a_{i}\right)=$ $\frac{1}{2}\left(F\left(a_{i}+0\right)+F\left(a_{i}-0\right)\right)$.

(B) There exists a number $b>0$ such that $F(x)$ and $F^{\prime}(x)$ are $O\left(e^{-\left(\frac{1}{2}+b\right)|x|}\right)$ as $|x| \rightarrow \infty$.

Then the Mellin transform of $F$,

$$
\Phi(s)=\int_{-\infty}^{+\infty} F(x) e^{\left(s-\frac{1}{2}\right) x} d x
$$

is holomorphic in every vertical strip $-a \leq \sigma \leq$ $1+a$ where $0<a<b, a<1$, and the sum $\sum \Phi(\rho)$ running over the non trivial zeros $\rho=\beta+i \gamma$ of $L(s, \chi)$ with $|\gamma|<T$ tends to a limit as $T$ tends to infinity. This limit is given by

$$
\begin{aligned}
\lim _{T \rightarrow+\infty} \sum_{|\gamma|<T} & \Phi(\rho) \\
=\ln \mathfrak{f}_{\chi}- & \sum_{\mathfrak{p}, m} \frac{\ln N_{K / \mathbb{Q}}(\mathfrak{p})}{N_{K / \mathbb{Q}}(\mathfrak{p})^{m / 2}} \chi^{\prime}(\mathfrak{p})^{m} F\left(m \ln N_{K / \mathbb{Q}}(\mathfrak{p})\right) \\
& -2(\ln 2 \pi+\gamma+2 \ln 2)-2 \varepsilon J(F)+2 I(F),
\end{aligned}
$$

where

$$
\begin{aligned}
& J(F)=\int_{0}^{+\infty} \frac{F(x)}{2 \cosh (x / 2)} d x, \\
& I(F)=\int_{0}^{+\infty} \frac{1-F(x)}{2 \sinh (x / 2)} d x,
\end{aligned}
$$

$\gamma=0.57721566 \ldots$ is the Euler constant and $\varepsilon$ is defined by Theorem 3.6.

\section{A. The Conditional Case}

Now we assume the Generalized Riemann Hypothesis $(\mathrm{GRH})$ for $L(s, \chi)$ which asserts that all the nontrivial zeros of $L(s, \chi)$ lie on the critical line $\operatorname{Re} s=\frac{1}{2}$. Now we write Theorem 4.1 for Serre's choice $F_{y}(x)=e^{-y x^{2}}(y>0)$. The Mellin transform $\Phi(s)$ of $F_{y}$ is

$$
\Phi_{y}(s)=\sqrt{\frac{\pi}{y}} e^{\left(s-\frac{1}{2}\right)^{2} /(4 y)},
$$

and the Fourier transform $\hat{F}_{y}$ of $F_{y}$ is

$$
\hat{F}_{y}(t)=\sqrt{\frac{\pi}{y}} e^{-t^{2} /(4 y)} .
$$

If we assume the GRH for $L(s, \chi)$, we can write $\Phi_{y}(\rho)=\hat{F}_{y}(t)$ where $\rho=\frac{1}{2}+i t$. For every $k \geq 1$, we denote by $t_{k}$ the positive imaginary part of the $k$-th zero of the Artin $L$-function $L(s, \chi)$, and $n_{k}$ its multiplicity. We have the identity

$$
\begin{aligned}
& S(y)=n_{\chi}+2 \sum_{k \geq 2}^{+\infty} n_{k} e^{-\frac{t_{k}^{2}}{4 y}} \\
&=-\sqrt{\frac{y}{\pi}} \sum_{\mathfrak{p}, m} \frac{\ln N_{K / \mathbb{Q}}(\mathfrak{p})}{N_{K / \mathbb{Q}}(\mathfrak{p})^{m / 2}} \chi^{\prime}(\mathfrak{p})^{m} e^{-y\left(m \ln N_{K / \mathbb{Q}}(\mathfrak{p})\right)^{2}} \\
&+\sqrt{\frac{y}{\pi}}\left(\ln \mathfrak{f}_{\chi}-2(\ln 2 \pi+\gamma+2 \ln 2)\right. \\
&\left.-2 \varepsilon J\left(F_{y}\right)+2 I\left(F_{y}\right)\right) .
\end{aligned}
$$

To compute $n_{\chi}$, one needs:

Proposition 4.2. Assuming the GRH, we have

$$
n_{\chi} \leq S(y) \quad \text { and } \quad \lim _{y \rightarrow 0} S(y)=n_{\chi}
$$

for all $y>0$.

The advantage of Serre's choice in Weil's explicit formula is that the series $S(y)$ converges rapidly to $n_{\chi}$ when $y \rightarrow 0$. In practice we prove for many quaternion fields that when $W(\chi)=+1$, we have $n_{\chi} \leq S(y)<2$ for some $y>0$ and so $n_{\chi}=0$. Similarly for $W(\chi)=-1$, we can prove the inequality $n_{\chi} \leq S(y)<3$ for some $y>0$ and so $n_{\chi}=1$. Actually, using Theorem 3.8, Conjecture 3.9 can be stated thus:

Proposition 4.3. Under GRH, Conjecture 3.9 holds if and only if there exists $y>0$ such that $S(y)<2$.

\section{B. The Unconditional Case}

The unconditional bounds of $n_{\chi}$ are less good than the GRH ones in Proposition 4.2 because of the requirement that $\operatorname{Re} \Phi(s) \geq 0$ on the whole critical strip. By using an argument of Odlyzko [Poitou 1977], this last condition holds when we take in Theorem 4.1 the function $G_{y}(x)=F_{y}(x) / \cosh (x / 2)$ with $F_{y}(x)=e^{-y x^{2}}(y>0)$. Thus we obtain the following bound of $n_{\chi}$.

Theorem 4.4. For all $y>0$, we have $n_{\chi} \leq T(y)$, where

$$
\begin{aligned}
T(y)= & \left(2 \int_{0}^{+\infty} \frac{e^{-y x^{2}}}{\cosh (x / 2)} d x\right)^{-1} \times \\
\left(\ln \mathfrak{f}_{\chi}\right. & -2 \sum_{\mathfrak{p}, m} \frac{\ln N_{K / \mathbb{Q}}(\mathfrak{p})}{1+N_{K / \mathbb{Q}}(\mathfrak{p})^{m}} \chi^{\prime}(\mathfrak{p})^{m} e^{-y\left(m \ln N_{K / \mathbb{Q}}(\mathfrak{p})\right)^{2}} \\
& \left.-2(\ln 2 \pi+\gamma+2 \ln 2)-2 \varepsilon J\left(G_{y}\right)+2 I\left(G_{y}\right)\right) .
\end{aligned}
$$


In practice we check Conjecture 3.9 using this criterion:

Proposition 4.5. Conjecture 3.9 holds if there exists $y>0$ such that $T(y)<2$.

To compute $S(y)$ and $T(y)$, we begin by computing the integrals $I\left(F_{y}\right), J\left(F_{y}\right), I\left(G_{y}\right)$ and $J\left(G_{y}\right)$ to a high enough precision, we then compute the series over the prime ideals in the Weil explicit formula by computing $\chi^{\prime}(\mathfrak{p})$ and $N_{K / \mathbb{Q}}(\mathfrak{p})$ for each prime number $p$ less than some large enough $p_{0}$. Actually the number field $N$ is defined by a polynomial $P(x)$; for every prime number $p$ prime to the index of $N$, the decomposition of the ideal $(p)$ into a product of prime ideals of $N$ is given by the decomposition of $P(x)$ modulo $p$; see [Cohen 1993]. Since $N / \mathbb{Q}$ is a Galois extension, then one needs to compute only the degree $f$ of the first irreducible polynomial appearing in the decomposition of $P(x)$ modulo $p$. The computations of $\chi^{\prime}(\mathfrak{p})$ and $N_{K / \mathbb{Q}}(\mathfrak{p})$ are given in the proposition below:

Proposition 4.6. Let $k_{1}=\mathbb{Q}\left(\sqrt{d_{1}}\right), k_{2}=\mathbb{Q}\left(\sqrt{d_{2}}\right)$, $k_{3}=\mathbb{Q}\left(\sqrt{d_{3}}\right)$ be the quadratic subfields of $N$.

- If $f=1$ then $N_{K / \mathbb{Q}}(\mathfrak{p})=p$ and $\chi^{\prime}(\mathfrak{p})=+1$.

- If $f=4$ then $N_{K / \mathbb{Q}}(\mathfrak{p})=p^{2}$ and $\chi^{\prime}(\mathfrak{p})=-1$.

- If $f=2$ we have two cases:

- If $\left(\frac{d_{i}}{p}\right)=-1$ for exactly one $i \in\{1,2,3\}$, then $N_{K / \mathbb{Q}}(\mathfrak{p})=p^{2}$ and $\chi^{\prime}(\mathfrak{p})=+1$.

- If $\left(\frac{d_{i}}{p}\right)=+1$ for exactly one $i \in\{1,2,3\}$, then $N_{K / \mathbb{Q}}(\mathfrak{p})=p$ and $\chi^{\prime}(\mathfrak{p})=-1$.

Example 4.7. Let $N=\mathbb{Q}(\sqrt{M})$, where

$$
M=\frac{5+\sqrt{5}}{2} \frac{21+\sqrt{21}}{2} .
$$

The quaternion field $N$ could be defined by the polynomial $P(x)$ in example 1 of section 6 . One can compute the different terms in $T(y)$ for $y=0.04$ and show that the sum over the prime ideals is equal to $-0.33763, J\left(G_{y}\right)=0.89478$ and $I\left(G_{y}\right)=0.83304$. Thus $T(y)=0.39377$.

When the conductor $\mathfrak{f}_{\chi}$ is large, the computation of $S(y)$ and $T(y)$ is slower and this is essentially due to the possible existence of low zeros of the Artin $L$-function $L(s, \chi)$. Actually when the first zeros of $L(s, \chi)$ distinct from $\frac{1}{2}$ are close to the real axis, one needs to compute $S(y)$ and $T(y)$ for smaller positive values of $y$ in order to be able to bound $S(y)$ and $T(y)$ above by 2 (see Propositions 4.3 and 4.5). An approach to the problem of low zeros of $L(s, \chi)$ in terms of the conductor $\mathfrak{f}_{\chi}$ is given in the next section.

\section{AN UPPER BOUND FOR $\mathrm{n}_{\chi}$ AND LOW ZEROS OF $\mathrm{L}(\mathrm{s}, \chi)$}

We now give estimates on the upper bounds of $n_{\chi}$ and the first zero $\rho_{\chi}=\frac{1}{2}+i \beta_{\chi}$ of $L(s, \chi)$ distinct from $\frac{1}{2}$. For this purpose, we apply Theorem 4.1 to suitable functions with compact supports. If we assume the GRH, then one can prove more precise estimates on $n_{\chi}$ and $\beta_{\chi}$. Such improvements have been considered in [Mestre 1986] for $L$-series of modular forms.

Theorem 5.1. Under GRH,

$$
n_{\chi} \ll \frac{\ln \mathfrak{f}_{\chi}}{\ln \ln \mathfrak{f}_{\chi}} \quad \text { and } \quad\left|\beta_{\chi}\right| \ll \frac{1}{\ln \ln \mathfrak{f}_{\chi}} .
$$

Proof. We first need an estimate for the sum over the prime ideals of $K$ in Theorem 4.1. Let $F$ be a function with compact support satisfying the hypotheses of Theorem 4.1 and let $F_{T}(x)=F\left(\frac{x}{T}\right)$. By using the prime number theorem, one can prove the following estimate:

Lemma 5.2. The sum over the prime ideals in Theorem 4.1 is bounded by the inequality

$\left|\sum_{\mathfrak{p}, m} \frac{\ln N_{K / \mathbb{Q}}(\mathfrak{p})}{N_{K / \mathbb{Q}}(\mathfrak{p})^{m / 2}} \chi^{\prime}(\mathfrak{p})^{m} F_{T}\left(m \ln N_{K / \mathbb{Q}}(\mathfrak{p})\right)\right| \leq C_{0} e^{T / 2}$, with $C_{0}>0$.

We also need an easy lemma:

Lemma 5.3. Define $F$ by

$$
F(x)= \begin{cases}1-|x| & \text { if }|x| \leq 1, \\ 0 & \text { otherwise. }\end{cases}
$$

Then F satisfies the hypotheses of Theorem 4.1 and

$$
\hat{F}(u)=\left(\frac{2 \sin (u / 2)}{u}\right)^{2} .
$$

Now if we put $F_{T}(x)=F\left(\frac{x}{T}\right)$ then $\hat{F}_{T}(u)=T \hat{F}(T u)$. Applying Weil's explicit formula to $F_{T}$ and using Lemma 5.2, we obtain the estimate:

$$
n_{\chi} T \leq \ln \mathfrak{f}_{\chi}+C_{0} e^{T / 2}+2\left(I\left(F_{T}\right)+J\left(F_{T}\right)\right),
$$


since $I\left(F_{T}\right)$ and $J\left(F_{T}\right)$ are bounded as $T$ tends to $+\infty$, replacing $T$ by $2 \ln \ln \mathfrak{f}_{\chi}$, we see that

$$
n_{\chi} \ll \frac{\ln \mathfrak{f}_{\chi}}{\ln \ln \mathfrak{f}_{\chi}},
$$

proving the first inequality in the statement of Theorem 5.1. To prove the theorem's second inequality, we use another even function $G$ with compact support, defined as follows.

Lemma 5.4. Let

$G(x)= \begin{cases}(1-x) \cos (\pi x)+\frac{3}{\pi} \sin (\pi x) & \text { if } 0 \leq x \leq 1 \\ 0 & \text { otherwise. }\end{cases}$

Then $G$ satisfies the hypotheses of Theorem 4.1 and

$$
\hat{G}(u)=\left(2-\frac{u^{2}}{\pi^{2}}\right)\left(\frac{2 \pi}{\pi^{2}-u^{2}} \cos \frac{u}{2}\right)^{2} .
$$

We now apply once more Weil's explicit formula to $G_{T}(x)=G(x / T)$ and replace $T$ by $\sqrt{2} \pi /\left|\beta_{\chi}\right|$. We obtain the estimate

$$
\begin{aligned}
\frac{8}{\pi^{2}} n_{\chi} T \geq & \ln \mathfrak{f}_{\chi}-2(\ln 2 \pi+\gamma+2 \ln 2) \\
& -2 \varepsilon J\left(G_{T}\right)+2 I\left(G_{T}\right) \\
& -\sum_{\mathfrak{p}, m} \frac{\ln N_{K / \mathbb{Q}}(\mathfrak{p})}{N_{K / \mathbb{Q}}(\mathfrak{p})^{m / 2}} \chi^{\prime}(\mathfrak{p})^{m} G_{T}\left(m \ln N_{K / \mathbb{Q}}(\mathfrak{p})\right) .
\end{aligned}
$$

Using Lemma 5.2, the above estimate (1) on $n_{\chi}$ and the fact that the integrals $I\left(G_{T}\right)$ and $J\left(G_{T}\right)$ are bounded as $T$ tends to $+\infty$, we deduce, for some positive constants $A$ and $B$ :

$$
\frac{\ln \mathfrak{f}_{\chi}}{\ln \ln \mathfrak{f}_{\chi}} A T+B e^{T / 2} \geq \ln \mathfrak{f}_{\chi},
$$

so that

$$
T \geq \min \left(\frac{1}{2 A}, 1-\frac{\ln (2 B)}{\ln \ln \mathfrak{f}_{\chi}}\right) \ln \ln \mathfrak{f}_{\chi} .
$$

Thus for sufficiently large $\mathfrak{f}_{\chi}$ we have $T \gg \ln \ln \mathfrak{f}_{\chi}$, and so

$$
\left|\beta_{\chi}\right| \ll \frac{1}{\ln \ln \mathfrak{f}_{\chi}},
$$

concluding the proof of the theorem.

Corollary 5.5. If we assume the GRH,

$$
\lim _{\mathfrak{f}_{\chi} \rightarrow+\infty} \rho_{\chi}=\frac{1}{2} .
$$

Without assuming the GRH, we have the following estimate for $n_{\chi}$, which is less good than the one in Theorem 5.1; see [Mestre 1983] for a similar result in the case of elliptic curves.
Theorem 5.6. $n_{\chi}<\ln \mathfrak{f}_{\chi}$ unconditionally.

Proof. Define the function $H_{T}$ with compact support by $H_{T}(x)=F_{T}(x) / \cosh (x / 2)$, where $F_{T}$ is defined after Lemma 5.3. By using an argument of Odlyzko [Poitou 1977], one can show that the Mellin transform $\Phi_{T}$ of $H_{T}$ satisfies $\operatorname{Re} \Phi_{T}(s) \geq 0$ in the critical strip. Thus, when we apply Theorem 4.1 to $H_{T}$, we obtain

$$
\begin{aligned}
n_{\chi} \Phi_{T}\left(\frac{1}{2}\right) & \\
\leq & \ln \mathfrak{f}_{\chi}-2(\ln 2 \pi+\gamma+2 \ln 2) \\
& -2 \varepsilon J\left(H_{T}\right)+2 I\left(H_{T}\right) \\
& -\sum_{\mathfrak{p}, m} \frac{\ln N_{K / \mathbb{Q}}(\mathfrak{p})}{N_{K / \mathbb{Q}}(\mathfrak{p})^{m / 2}} \chi^{\prime}(\mathfrak{p})^{m} H_{T}\left(m \ln N_{K / \mathbb{Q}}(\mathfrak{p})\right) .
\end{aligned}
$$

Since $H_{T}$ is a decreasing function on $[0,+\infty]$, one can show:

Lemma 5.7.

$$
\begin{aligned}
\left|\sum_{\mathfrak{p}, m} \frac{\ln N_{K / \mathbb{Q}}(\mathfrak{p})}{N_{K / \mathbb{Q}}(\mathfrak{p})^{m / 2}} \chi^{\prime}(\mathfrak{p})^{m} H_{T}\left(m \ln N_{K / \mathbb{Q}}(\mathfrak{p})\right)\right| \\
\leq 4 \sum_{p^{m} \leq e^{T}} \frac{\ln p}{p^{m / 2}} H_{T}(m \ln p) .
\end{aligned}
$$

Thus, by using the inequality before the lemma, we obtain

$$
\begin{aligned}
n_{\chi} \Phi_{T}\left(\frac{1}{2}\right) \leq & \ln \mathfrak{f}_{\chi}-2(\ln 4 \pi+\gamma)+2 J\left(H_{T}\right)+2 I\left(H_{T}\right) \\
& +4 \sum_{p^{m} \leq e^{T}} \frac{\ln p}{p^{m / 2}} H_{T}(m \ln p) .
\end{aligned}
$$

Now if we put $T=\ln 3$, we obtain

$$
\begin{aligned}
1.072 n_{\chi} & \leq \ln \mathfrak{f}_{\chi}-6.216+0.523+4.648+0.683 \\
& \leq \ln \mathfrak{f}_{\chi}-0.362 .
\end{aligned}
$$

And so we find that $n_{\chi}<\ln \mathfrak{f}_{\chi}$.

\section{COMPUTATIONS OF $\mathrm{n}_{\chi}$ FOR QUATERNION FIELDS}

Table 1 gives our computed data. Each box refers to one quaternion field $N / \mathbb{Q}$, giving on the top line a reduced polynomial $P(x)$ ("reduced" meaning that we have written $N=\mathbb{Q}[\theta]$, choosing for $\theta$ a minimal primitive vector of the lattice of integers of $N$ for the "twisted" trace form $\operatorname{tr}_{N / \mathbb{Q}}(x \bar{y})$ ), and on the bottom line other related information. The computations were done using PARI-GP version 2.0.19.

According to [Kwon 1996], the minimum discriminant both in the real and in the imaginary case is 


\begin{tabular}{|c|c|c|c|c|c|c|c|c|c|}
\hline & $P(x)$ and $D_{N}$ & $\mathrm{R} / \mathrm{I}$ & quad. subfields & $W(\chi)$ & $y_{0}$ & $S\left(y_{0}\right)$ & $y$ & $T(y)$ & $n_{\chi}$ \\
\hline \multirow{2}{*}{1} & \multicolumn{9}{|c|}{$x^{8}-x^{7}-34 x^{6}+29 x^{5}+361 x^{4}-305 x^{3}-1090 x^{2}+1345 x-395$} \\
\hline & 1340095640625 & $\mathrm{R}$ & $\mathbb{Q}(\sqrt{5}), \mathbb{Q}(\sqrt{21})$ & +1 & 0.04 & 0.00806 & 0.04 & 0.393771 & 0 \\
\hline \multirow{2}{*}{2} & \multicolumn{9}{|c|}{$x^{8}+315 x^{6}+34020 x^{4}+1488375 x^{2}+22325625$} \\
\hline & 1340095640625 & I & $\mathbb{Q}(\sqrt{5}), \mathbb{Q}(\sqrt{21})$ & -1 & 0.07 & 1.04505 & 0.11 & 1.58039 & 1 \\
\hline \multirow{2}{*}{3} & \multicolumn{9}{|c|}{$x^{8}-205 x^{6}+13940 x^{4}-378225 x^{2}+3404025$} \\
\hline & 74220378765625 & $\mathrm{R}$ & $\mathbb{Q}(\sqrt{5}), \mathbb{Q}(\sqrt{41})$ & -1 & 0.05 & 1.00067 & 0.1 & 1.30413 & 1 \\
\hline \multirow{2}{*}{4} & \multicolumn{9}{|c|}{$x^{8}-3 x^{7}+142 x^{6}-115 x^{5}+6641 x^{4}+3055 x^{3}+157938 x^{2}+152941 x+2031361$} \\
\hline & 6011850680015625 & I & $\mathbb{Q}(\sqrt{5}), \mathbb{Q}(\sqrt{41})$ & -1 & 0.05 & 1.26425 & 0.05 & 2.09134 & 1 \\
\hline \multirow{2}{*}{5} & \multicolumn{9}{|c|}{$x^{8}-x^{7}-178 x^{6}-550 x^{5}+7225 x^{4}+44407 x^{3}+55928 x^{2}-45392 x+4096$} \\
\hline & 31172897213027361 & $\mathrm{R}$ & $\mathbb{Q}(\sqrt{17}), \mathbb{Q}(\sqrt{33})$ & +1 & 0.04 & 0.00222 & 0.04 & 0.31774 & 0 \\
\hline \multirow{2}{*}{6} & \multicolumn{9}{|c|}{$x^{8}-3 x^{7}+106 x^{6}+381 x^{5}+414 x^{4}-8475 x^{3}+44497 x^{2}+151740 x+253168$} \\
\hline & 31172897213027361 & I & $\mathbb{Q}(\sqrt{17}), \mathbb{Q}(\sqrt{33})$ & -1 & 0.04 & 1.19064 & 0.04 & 2.05980 & 1 \\
\hline \multirow{2}{*}{7} & \multicolumn{9}{|c|}{$x^{8}-3 x^{7}-475 x^{6}-2386 x^{5}+56669 x^{4}+732202 x^{3}+3280440 x^{2}+5788174 x+2396941$} \\
\hline & 12187467896636600569 & $\mathrm{R}$ & $\mathbb{Q}(\sqrt{37}), \mathbb{Q}(\sqrt{41})$ & -1 & 0.03 & 1.03133 & 0.03 & 1.75340 & 1 \\
\hline \multirow{2}{*}{8} & \multicolumn{9}{|c|}{$x^{8}-3 x^{7}-847 x^{6}-4250 x^{5}+194805 x^{4}+2321042 x^{3}+4218300 x^{2}-28827252 x-48031623$} \\
\hline & 388282220975269366201 & $\mathrm{R}$ & $\mathbb{Q}(\sqrt{37}), \mathbb{Q}(\sqrt{73})$ & -1 & 0.03 & 1.00010 & 0.03 & 1.35751 & 1 \\
\hline \multirow{2}{*}{9} & \multicolumn{9}{|c|}{$x^{8}-3 x^{7}+1854 x^{6}+14657 x^{5}+1134753 x^{4}+15385779 x^{3}+370857442 x^{2}+2861780247 x+28470071727$} \\
\hline & 31450859898996818662281 & I & $\mathbb{Q}(\sqrt{37}), \mathbb{Q}(\sqrt{73})$ & -1 & 0.03 & 1.84217 & 0.01 & 2.83822 & 1 \\
\hline \multirow{2}{*}{10} & \multicolumn{9}{|c|}{$x^{8}-3 x^{7}+1042 x^{6}+8233 x^{5}+284219 x^{4}+4899401 x^{3}+42209694 x^{2}+179998937 x+404059099$} \\
\hline & 987184899627564646089 & I & $\mathbb{Q}(\sqrt{37}), \mathbb{Q}(\sqrt{41})$ & -1 & 0.03 & 1.58551 & 0.03 & 2.81849 & 1 \\
\hline \multirow{2}{*}{11} & \multicolumn{9}{|c|}{$x^{8}-x^{7}-866 x^{6}-2686 x^{5}+197617 x^{4}+1072207 x^{3}-8786448 x^{2}-32864208 x+159160192$} \\
\hline & 420386522758923179809 & $\mathrm{R}$ & $\mathbb{Q}(\sqrt{17}), \mathbb{Q}(\sqrt{161})$ & +1 & 0.03 & 0.19296 & 0.03 & 1.13789 & 0 \\
\hline \multirow{2}{*}{12} & \multicolumn{9}{|c|}{$x^{8}-3 x^{7}-1591 x^{6}-7978 x^{5}+718061 x^{4}+8174530 x^{3}-29006964 x^{2}-433628432 x+235862473$} \\
\hline & 16964214194699233633081 & $\mathrm{R}$ & $\mathbb{Q}(\sqrt{37}), \mathbb{Q}(\sqrt{137})$ & -1 & 0.03 & 1.00204 & 0.03 & 1.64797 & 1 \\
\hline \multirow{2}{*}{13} & \multicolumn{9}{|c|}{$x^{8}-3 x^{7}+3478 x^{6}+27505 x^{5}+4489397 x^{4}+53881703 x^{3}+2972520282 x^{2}+26220344507 x+651061429207$} \\
\hline & 1374101349770637924279561 & I & $\mathbb{Q}(\sqrt{37}), \mathbb{Q}(\sqrt{137})$ & -1 & 0.05 & 2.24737 & 0.01 & 2.88613 & 1 \\
\hline 14 & \multicolumn{9}{|l|}{$x^{8}-12 x^{6}+36 x^{4}-36 x^{2}+9$} \\
\hline 14 & 12230590464 & $\mathrm{R}$ & $\mathbb{Q}(\sqrt{2}), \mathbb{Q}(\sqrt{3})$ & +1 & 0.05 & 0.00002 & 0.08 & 0.11665 & 0 \\
\hline 15 & $x^{8}+12 x^{6}+36 x^{4}+36 x^{2}+9$ & & & & & & & & \\
\hline 15 & 12230590464 & I & $\mathbb{Q}(\sqrt{2}), \mathbb{Q}(\sqrt{3})$ & & 0.05 & 1.000005 & 0.05 & 1.05777 & $\leq 1$ \\
\hline 16 & $x^{8}-44 x^{6}+308 x^{4}-484 x^{2}+12$ & & & & & & & & \\
\hline 10 & 29721861554176 & $\mathrm{R}$ & $\mathbb{Q}(\sqrt{2}), \mathbb{Q}(\sqrt{11})$ & +1 & 0.05 & 0.01167 & 0.05 & 0.36928 & 0 \\
\hline 17 & $x^{8}-76 x^{6}+1748 x^{4}-12996 x^{2}+$ & 2924 & & & & & & & \\
\hline 17 & 789298907447296 & $\mathrm{R}$ & $\mathbb{Q}(\sqrt{2}), \mathbb{Q}(\sqrt{19})$ & +1 & 0.04 & 0.04449 & 0.04 & 0.66149 & 0 \\
\hline
\end{tabular}

TABLE 1 (start). For each quaternion field $N / \mathbb{Q}$, we show a reduced polynomial $P(x)$ (see beginning of Section 6), the discriminant $d_{N}$, whether $N$ is real or imaginary, two quadratic subfields $\mathbb{Q}\left(\sqrt{d_{1}}\right)$ and $\mathbb{Q}\left(\sqrt{d_{2}}\right)$ of $N$ - the third being $\mathbb{Q}\left(\sqrt{d_{1} d_{2}}\right)$ - and the values of $W(\chi), y_{0}, S\left(y_{0}\right)$ (Proposition 4.3), y, T(y) (Proposition 4.5) and $n_{\chi}$. 


\begin{tabular}{|c|c|c|c|c|c|c|c|c|c|}
\hline & $P(x)$ and $D_{N}$ & $\mathrm{R} / \mathrm{I}$ & quad. subfields & $W(\chi)$ & $y_{0}$ & $S\left(y_{0}\right)$ & $y$ & $T(y)$ & $n_{\chi}$ \\
\hline \multirow{2}{*}{18} & \multicolumn{9}{|c|}{$x^{8}-60 x^{6}+810 x^{4}-1800 x^{2}+900$} \\
\hline & 47775744000000 & $\mathrm{R}$ & $\mathbb{Q}(\sqrt{5}), \mathbb{Q}(\sqrt{6})$ & & 0.07 & 1.00101 & 0.07 & 1.13852 & $\leq 1$ \\
\hline \multirow{2}{*}{19} & \multicolumn{9}{|c|}{$x^{8}-60 x^{6}+1170 x^{4}-9000 x^{2}+22500$} \\
\hline & 47775744000000 & $\mathrm{R}$ & $\mathbb{Q}(\sqrt{5}), \mathbb{Q}(\sqrt{6})$ & +1 & 0.07 & 0.09399 & 0.07 & 0.61520 & 0 \\
\hline \multirow{2}{*}{20} & \multicolumn{9}{|c|}{$x^{8}+60 x^{6}+810 x^{4}+1800 x^{2}+900$} \\
\hline & 47775744000000 & I & $\mathbb{Q}(\sqrt{5}), \mathbb{Q}(\sqrt{6})$ & & 0.07 & 1.07405 & 0.07 & 1.55366 & $\leq 1$ \\
\hline \multirow{2}{*}{21} & \multicolumn{9}{|c|}{$x^{8}+60 x^{6}+1170 x^{4}+9000 x^{2}+22500$} \\
\hline & 47775744000000 & I & $\mathbb{Q}(\sqrt{5}), \mathbb{Q}(\sqrt{6})$ & & 0.08 & 1.09340 & 0.07 & 1.63606 & $\leq 1$ \\
\hline \multirow{2}{*}{22} & \multicolumn{9}{|c|}{$x^{8}+105 x^{6}+3780 x^{4}+55125 x^{2}+275625$} \\
\hline & 343064484000000 & I & $\mathbb{Q}(\sqrt{5}), \mathbb{Q}(\sqrt{21})$ & +1 & 0.05 & 0.54966 & 0.05 & 1.53349 & 0 \\
\hline \multirow{2}{*}{23} & \multicolumn{9}{|c|}{$x^{8}+205 x^{6}+13940 x^{4}+378225 x^{2}+3404025$} \\
\hline & 19000416964000000 & I & $\mathbb{Q}(\sqrt{5}), \mathbb{Q}(\sqrt{41})$ & & 0.05 & 1.13981 & 0.03 & 1.80213 & $\leq 1$ \\
\hline
\end{tabular}

TABLE 1 (continued)

$2^{24} 3^{6}$, attained exactly in the fields 14 and 15 ; similarly the smallest coincidences between two real or imaginary fields occur for the discriminant $2^{22} 5^{6} 3^{6}$, attained exacltly on the four fields 18 to 21 . Fields 1 to 13 are tame, the others are not.

\section{ACKNOWLEDGMENTS}

I thank J. Martinet for bringing this problem to my attention and for his helpful comments and suggestions. I also profited from discussions with $\mathrm{H}$. Cohen and B. Erez. I also thank J.-P. Serre for his remarks on an earlier version of this paper.

\section{REFERENCES}

[Barner 1981] K. Barner, "On A. Weil's explicit formula", J. Reine Angew. Math. 323 (1981), 139152.

[Cohen 1993] H. Cohen, A course in computational algebraic number theory, Graduate Texts in Math. 138, Springer, Berlin, 1993.

[Cohen 2000] H. Cohen, Advanced topics in computational number theory, Graduate Texts in Math. 193, Springer, New York, 2000.

[Damey and Payan 1970] P. Damey and J.-J. Payan, "Existence et construction des extensions galoisiennes et non-abéliennes de degré 8 d'un corps de caractéristique différente de 2", J. Reine Angew. Math. 244 (1970), 37-54.
[Fröhlich 1972] A. Fröhlich, "Artin root numbers and normal integral bases for quaternion fields", Invent. Math. 17 (1972), 143-166.

[Goss 1996] D. Goss, Basic structures of function field arithmetic, Ergebnisse der Math. (3) 35, Springer, Berlin, 1996.

[Kwon 1996] S.-H. Kwon, "Sur les discriminants minimaux des corps quaternioniens", Arch. Math. (Basel) 67:2 (1996), 119-125.

[Martinet 1971] J. Martinet, "Modules sur l'algèbre du groupe quaternionien", Ann. Sci. École Norm. Sup. (4) 4 (1971), 399-408.

[Martinet 1977] J. Martinet, "Character theory and Artin L-functions", pp. 1-87 in Algebraic number fields: L-functions and Galois properties (Durham, Durham, 1975), edited by A. Fröhlich, Academic Press, London, 1977.

[Mestre 1983] J.-F. Mestre, "Courbes elliptiques et formules explicites", pp. 179-187 in Séminaire de théorie des nombres (Paris, 1981/1982), Progress in Math. 38, Birkhäuser Boston, Boston, MA, 1983.

[Mestre 1986] J.-F. Mestre, "Formules explicites et minorations de conducteurs de variétés algébriques", Compositio Math. 58:2 (1986), 209-232.

[Murty and Murty 1997] M. R. Murty and V. K. Murty, Non-vanishing of L-functions and applications, Birkhäuser, Basel, 1997. 
[Poitou 1977] G. Poitou, "Sur les petits discriminants", pp. Exp. No. 6, 18 in Séminaire Delange-PisotPoitou, 18e année (1976/77), Secrétariat Math., Paris, 1977.

[Serre 1970] J.-P. Serre, Cours d'arithmétique, Presses Universitaires de France, Paris, 1970.

[Serre 1978] J.-P. Serre, Représentations linéaires des groupes finis, revised ed., Hermann, Paris, 1978.

[Stark 1974] H. M. Stark, "Some effective cases of the Brauer-Siegel theorem", Invent. Math. 23 (1974), $135-152$.
[Tollis 1997] E. Tollis, "Zeros of Dedekind zeta functions in the critical strip", Math. Comp. 66:219 (1997), 1295-1321.

[Weil 1972] A. Weil, "Sur les formules explicites de la théorie des nombres", Izv. Akad. Nauk SSSR Ser. Mat. 36 (1972), 3-18. Reprinted as pp. 249-264 in his Euvres, v. 3, Springer, Heidelberg, 1979.

[Witt 1936] E. Witt, "Konstruktion von galoisschen Körpern der Charakteristik $p$ zu vorgegebener Gruppe der Ordnung $p^{f} "$, J. Reine angew. Math. 174 (1936), 237-245. Reprinted as pp. 120-128 in his Gesammelte Abhandlungen, Springer, Heidelberg, 1991.

Sami Omar, Université Bordeaux I, Laboratoire A2X, U.M.R. Université, CNRS numéro 5465 351, Cours de la Libération, F-33405 Talence, France (omar@math.u-bordeaux.fr)

Received February 14, 2000; accepted in revised form November 4, 2000 